\title{
Examining the triarchic model of psychopathy using revised Reinforcement Sensitivity Theory ${ }^{\text {is }}$
}

\author{
John J. Donahue ${ }^{\mathrm{a}, *}$, Leonardo J. Caraballo ${ }^{\mathrm{b}, *}$ \\ a Division of Applied Behavioral Sciences, University of Baltimore, LC 408, 1420 N. Charles St., Baltimore, MD 21201, United States \\ ${ }^{\mathrm{b}}$ Phoenix VA Health Care System, 650 E. Indian School Road, Phoenix, AZ 85012, United States
}

\section{A R T I C L E I N F O}

\section{Article history:}

Received 17 September 2014

Received in revised form 17 February 2015

Accepted 23 February 2015

\section{Keywords:}

Reinforcement Sensitivity Theory

Psychopathy

Triarchic model

\begin{abstract}
A B S T R A C T
Available research suggests the neurobiological systems reflected in Reinforcement Sensitivity Theory (RST) are important in etiological conceptualizations of psychopathy, however few studies have utilized the revised RST model (r-RST). Moreover, very little research has examined the associations between r-RST systems and the triarchic psychopathic constructs of boldness, meanness, and disinhibition. Using a recently developed measure of r-RST (Jackson Five; Jackson, 2009), the present study examined the triarchic conceptualization of psychopathy in relation to r-RST in a sample of 301 adult participants. Results suggest that the triarchic construct of boldness is characterized by increased r-BAS activity and deficits in r-FFFS activity; meanness is associated with increased r-BIS and r-Fight activity, and diminished r-BAS, r-Flight, and r-Freeze activity; and, disinhibition is characterized by increased r-BAS, r-Fight, and r-Freeze, with deficits in r-Flight. These results add to our understanding of how the triarchic constructs relate to r-FFFS and r-BAS, however they raise several questions concerning the construct validity of r-BIS as measured by the Jackson Five.
\end{abstract}

Published by Elsevier Ltd.

\section{Introduction}

Reinforcement Sensitivity Theory (RST) is a neurobiological account of the primary motivational systems proposed to underlie personality (Gray, 1970). Originally articulated by Gray, RST emphasized three major motivational systems underlying emotion, and variation in these systems were proposed to explain individual differences in personality and psychopathology. The FightFlight System (FFS) was hypothesized as sensitive to unconditioned aversive stimuli, the Behavioral Approach System (BAS) reflected sensitivities to conditioned appetitive stimuli associated with reward, and the Behavioral Inhibition System (BIS) was hypothesized to be sensitive to conditioned aversive stimuli which served as signals of punishment or termination of reward (Gray, 1982). The combined effects of punishment (BIS and FFS) and

\footnotetext{
Writing of this manuscript was largely conducted while the first author was a postdoctoral fellow at VISN 20 NW Mental Illness Research, Education and Clinical Center (MIRECC), Portland VA Medical Center, and was supported by the Office of Academic Affiliations, Advanced Fellowship Program in Mental Illness Research and Treatment, Department of Veterans Affairs.

* Corresponding authors. Tel.: +1 (410) 8375831 (J.J. Donahue).

E-mail addresses: jdonahue@ubalt.edu (J.J. Donahue), Leonardo.Caraballo@va. gov (L.J. Caraballo).
}

reward (BAS) sensitivity are considered causally related to phenotypic expressions of extraversion and neuroticism (Corr, DeYoung, \& McNaughton, 2013).

RST underwent a major revision in 2000 (r-RST; Gray \& McNaughton, 2000), in which alterations were made to the hypothesized stimuli that provide input into the three systems. Under r-RST, r-BAS is now hypothesized to respond to all appetitive stimuli (conditioned and unconditioned), and is mainly related to extraversion (Smillie, Pickering, \& Jackson, 2006). The freeze response was incorporated into FFS, and the Fight-Flight-Freeze System (r-FFFS) is now hypothesized to mediate responses to both unconditioned and conditioned aversive stimuli and the experience of fear. Finally, r-BIS is now considered a regulatory mechanism for goal conflicts rather than solely mediating reactions to conditioned aversive stimuli (Gray \& McNaughton, 2000). Specifically, r-BIS activation includes risk assessment and goal conflict resolution in the context of both BAS and FFFS activation, and is subjectively experienced as worry and anxious apprehension. One can see that changes to r-RST largely related to the distinction between conditioned and unconditioned stimuli, as this division was no longer considered relevant. Additionally, substantial research has demonstrated a distinction between the emotions of fear and anxiety (see Barlow, 2002), and the revised theory clearly reflects these advancements (Corr, 2008). 


\subsection{Psychopathy and RST}

Psychopathy is a personality disorder reflecting characteristics of behavioral deviancy, as well as deficits in emotional and interpersonal functioning (Cleckley, 1941). It is a construct that has been subject to widespread investigation of potential etiological mechanisms, perhaps more so than any other personality pathology. Accumulating evidence suggests that psychopathy is heterogeneous in nature (Skeem, Poythress, Edens, Lilienfeld, \& Cale, 2003), therefore attempts to refine our etiological understanding of this disorder must account for its phenotypic variation.

Karpman (1941) was among the first to theorize there may be distinct groupings of individuals with psychopathy, emphasizing a two subtype model. In his description of primary psychopathy, emotional and interpersonal deficits were considered core features. Secondary psychopathy, although behaviorally indistinguishable from primary psychopathy, was thought to be the result of impulsivity and emotional intolerance. The majority of research examining psychopathy within a RST framework has focused on the primary/secondary subtypes. Lykken (1995) suggested that the innately fearless temperament of primary psychopathy is reflective of a weak BIS, whereas secondary psychopathy may instead result from an overactive BAS. Supporting this hypothesis, in a sample of incarcerated inmates, Newman, MacCoon, Vaughn, and Sadeh (2005) found primary psychopathy to be characterized by a hypoactive BIS and normal BAS, while secondary psychopathy exhibited a hyperactive BAS and BIS. In a noninstitutionalized sample, Ross et al. (2007) found a hypoactive BIS to be associated with primary psychopathy, however a hyperactive BAS was associated with both primary and secondary variants. Consistent with this, a recent review on this topic reported a weak BIS appeared uniquely related to primary psychopathy, while an overactive BAS was associated with both primary and secondary subtypes (Bijttebier, Beck, Claes, \& Vandereycken, 2009).

Although RST was revised in 2000, the vast majority of studies on psychopathy have used the original RST framework. Carver and White's (1994) BIS/BAS Scales, based on the original RST, is the most commonly used self-report measure of sensitivities to reinforcement. The BIS/BAS Scales are comprised of four subscales, one corresponding to BIS, and three BAS scales (reward sensitivity, drive, and fun-seeking). However, r-RST no longer views fear sensitivity to be a function of the BIS. As such, the validity of this measure has been called into question as it conflates fear and anxiety under one BIS subscale (Poythress et al., 2008). In an attempt to address this discrepancy, Heym, Ferguson, and Lawrence (2008) demonstrated that the BIS scale is best represented as two factors composed of BIS-anxiety and BIS-fear, and that splitting the scale may be effective in gauging r-RST. Using this methodology, Hughes, Moore, Morris, and Corr (2012) demonstrated that BISanxiety was negatively associated with both primary and secondary psychopathic characteristics; BIS-fear was negatively correlated with only primary psychopathy; BAS-reward sensitivity and drive were positively related to primary psychopathy; and BAS-fun seeking was positively correlated with secondary psychopathy but negatively correlated with primary characteristics. In a follow-up study, BIS-anxiety and BIS-fear were negatively related to primary psychopathy, but positively related to secondary psychopathy (Broerman, Ross, \& Corr, 2014). Overall, while the method of splitting the BIS/BAS Scales is an important first step at examining the relationship between psychopathy and r-RST, this measure was not created to assess the revised constructs and replication using psychometrically valid measures of r-RST is necessary.

Recently, the Jackson Five Scales were developed to assess the relevant constructs of r-RST with separate subscales for r-BIS, rFight, r-Flight, r-Fear, and r-BAS (Jackson, 2009). In order to clearly differentiate r-BIS from r-FFFS by avoiding constructs related to fear, Jackson sought to operationalize r-BIS as social conflicts including defensive approach, social comparison, and effort failure (White \& Depue, 1999). During its preliminary validation (Jackson, 2009), the Jackson Five was compared to a short form of the Psychopathic Personality Inventory (PPI; Lilienfeld \& Andrews, 1996). In this study, r-BAS was positively associated with both the Fearless Dominance and Self-Centered Impulsivity factors of the PPI; r-BIS was negatively associated with Fearless Dominance and unrelated to Self-Centered Impulsivity; and, r-FFFS was negatively associated with Fearless Dominance but positively associated with Self-Centered Impulsivity. Since then however, there has been question about the breadth of content of the Jackson Five r-BIS scale, with suggestions that it appears to tap into the construct of social competitiveness, rather than BIS (Smederevac, Mitrović, Čolović, \& Nikolašević, 2014). As such, additional research examining the construct validity of the Jackson Five Scales is warranted.

\subsection{The triarchic conceptualization of psychopathy and r-RST}

Patrick and colleagues have recently proposed a triarchic conceptualization of psychopathy, in which the constructs of boldness, meanness, and disinhibition are suggested to underlie its presentation (Patrick, Fowles, \& Krueger, 2009), and variation in these constructs may explain differences in presentation. Boldness refers to characteristics of calm and poise in stressful situations, a high degree of uncertainty tolerance, and a socially dominant interpersonal style. Meanness describes deficiencies in empathy, a lack of close attachments, and a behavioral style that is exploitative and sensation-seeking. Finally, disinhibition reflects an externalizing style which includes impulse control problems, a lack of planfulness, difficulties with emotion regulation, and limited behavioral controls (i.e., the intersection of impulsivity and negative affect). The primary and secondary variants of psychopathy can therefore be thought to map on to the triarchic constructs, with primary psychopathy being preferentially characterized by boldness and low negative affect, secondary psychopathy characterized by disinhibition, and increased meanness common to both (see Drislane et al., 2014). Recent research examining the triarchic constructs in relation to o-RST in a mixed sample of incarcerated inmates and undergraduate students found o-BIS to be negatively associated with boldness and meanness, and o-BAS to be positively associated with boldness and disinhibition (Sellbom \& Phillips, 2013).

Several hypotheses can be made based on a theoretical understanding of the triarchic constructs and prior empirical research on RST and primary/secondary psychopathy. The present study therefore seeks to examine the triarchic conceptualization of psychopathy in relation to the constructs of r-RST, as measured by the Jackson Five. As boldness is considered to reflect the social dominance and emotional resilience (Patrick et al., 2009) characteristics of primary psychopathy, we hypothesized this factor would be positively associated with r-BAS, and negatively associated with r-FFFS and r-BIS. Given that Lykken's low-fear hypothesis of psychopathy may be better understood under r-RST as weak FFFS, rather than weak BIS (Poythress et al., 2008), we expected that boldness would exhibit a stronger association with overall $r$ FFFS as compared to BIS. Next, because impulsivity and negative emotionality underlies disinhibition (Patrick et al., 2009) and is prototypic of secondary psychopathy, we hypothesized this factor would be positively related to r-BAS, r-FFFS, and r-BIS. Finally, as meanness refers to a lack of empathy, an exploitative and cruel interpersonal style, and excitement seeking (Patrick et al., 2009), we expected this factor to be positively associated with r-BAS and negatively associated with r-BIS. While we further hypothesized an overall negative relationship with r-FFFS, we did expect 
meanness to be positively associated with the Fight domain of the r-FFFS.

\section{Method}

\subsection{Participants}

Participants were 301 undergraduate students (70\% female) at a liberal arts university who participated in exchange for course extra credit. Participant ages ranged from 18 to $67(M=21.33$, $S D=5.90, M d n=20.00$ ). Racial/ethnic composition of the sample included 64.3\% Caucasian, 16\% African-American, 9.3\% Hispanic, 5.3\% Asian, and 5\% from other racial backgrounds.

\subsection{Measures}

\subsubsection{Psychopathic Personality Inventory - Revised (PPI-R)}

The PPI-R (Lilienfeld \& Widows, 2005) is a 154-item self-report measure designed to assess global psychopathy, as well as its component traits, in both clinical and nonclinical settings. Items are rated on a 4-point Likert scale ranging from 1 (False) to 4 (True). The measure is comprised of eight factor-analytically derived subscales and three validity indices. The eight subscales largely map onto two higher-order factors, Fearless Dominance (FD) and SelfCentered Impulsivity (SCI), with the Coldheartedness subscale loading onto its own third factor (Lilienfeld \& Widows, 2005).

The triarchic constructs of Boldness, Meanness, and Disinhibition were indexed using PPI scales developed by Hall et al. (2014). Through a three phase process of scale development (item selection, refinement, and psychometric evaluation), PPI scales indexing boldness, meanness, and disinhibition were constructed and shown to demonstrate good internal consistency and construct validity in both student and forensic samples. While the majority of items on the PPI are also included in the PPI-R, seven items were omitted as they lacked a PPI-R counterpart. Analyses conducted by Hall and colleagues suggest this method results in negligible changes to internal consistency and correlations with external criteria. In this study, PPI-Boldness is therefore comprised of 23 items derived from the FD factor; PPIMeanness reflects 18 items derived from the Coldheartedness and Machiavellian Egocentricity subscales; and PPI-Disinhibition is made up of 18 items from the SCI factor (see Hall et al., 2014, for a list of PPI-R items comprising each scale). In measuring the triarchic scales, we chose to utilize the PPI-based triarchic scales rather than the PPI-R factors primarily due to the finding that the Coldheartedness subscale does not fully capture the Meanness construct (Hall et al., 2014). In the current study, internal consistencies (Cronbach's $\alpha$ ) for PPI-Boldness $(\alpha=.81$ ), PPI-Meanness $(\alpha=.76)$, and PPI-Disinhibition $(\alpha=.72)$ were adequate.

\subsubsection{Jackson Five Scales}

The Jackson Five (Jackson, 2009) is a 30 -item self-report measure of the constructs of revised Reinforcement Sensitivity Theory. It is comprised of three scales measuring the motivational systems of r-RST, specifically, the Behavioral Approach System ( $\mathrm{r}-$ BAS), the Behavioral Inhibition System (r-BIS), and the FightFlight-Freeze system (r-FFFS). The FFFS is also broken down into its component subscales of Fight, Flight and Freezing. In the current study, internal consistency was considered acceptable for the three primary scales of r-BIS $(\alpha=.62)$, r-BAS $(\alpha=.73)$, and rFFFS $(\alpha=.68)$. While the reliability coefficients for the subcomponents of FFFS were rather low ( $\alpha$ 's ranging from .58 to .78), they are consistent with previous research (Harnett, Loxton, \& Jackson, 2013).

\subsection{Procedure}

Participants completed paper-and-pencil questionnaires in small groups. This study received full approval from the University Institutional Review Board and informed consent was reviewed with participants prior to administration of questionnaires.

Prior to data analysis, 14 cases were excluded based on elevations on the Inconsistent Responding-15 scale of the PPI-R that are considered highly atypical $(\geqslant 17)$, resulting in a sample of 287 participants ( $70 \%$ female). Bivariate correlations were computed among PPI-R total and factor scores, PPI-R triarchic constructs, and the r-RST constructs of r-BAS, r-BIS, and the r-FFFS dimensions. Next, three separate hierarchical multiple regression analyses were conducted to examine the unique associations between r-RST variables and PPI-R triarchic variables (PPI-Boldness, PPI-Meanness, and PPI-Disinhibition). Age and sex were entered into the first step, and r-RST variables were entered into the second step. Given the expected divergent associations between the three subdomains of the r-FFFS and psychopathic traits, these variables (r-Fight, r-Flight, and r-Freeze) were entered individually rather than at the FFFS level of analysis.

\section{Results}

\subsection{Descriptive statistics}

Means, standard deviations, and zero-order correlations for psychopathy dimensions, r-RST constructs, and age are displayed in Table 1. At the bivariate level, PPI-Boldness exhibited a strong positive association with $r$-BAS $(r=.50)$, a moderate negative association with r-FFFS ( $r=-.33)$, and was unrelated to r-BIS $(r=.07$, $n s)$, partially supportive of our hypotheses. As expected, PPIDisinhibition exhibited positive associations with r-BAS $(r=.18)$ and $\mathrm{r}$-FFFS $(r=.27)$, although $\mathrm{r}$-BIS was unrelated with this variable $(r=.11, n s)$. The subcomponents of r-FFFS displayed some variability in their relationships with PPI-Disinhibition, with r-Fight $(r=.33)$ and $r$-Freeze $(r=.28)$ displaying moderately positively associations, and r-Flight demonstrating no significant association $(r=-.08, n s)$. Finally, contrary to hypotheses, PPI-Meanness exhibited a moderately positive association with $r$-BIS $(r=.24)$, and no significant associations with r-BAS or r-FFFS were found. As expected however, when examining the subcomponents of r-FFFS, PPI-Meanness was positively associated with r-Fight $(r=.33)$, but negatively associated with $r$-Flight $(r=-.24)$ and r-Freeze $(r=-.14)$.

\section{2. $r$-RST constructs predicting triarchic psychopathy constructs}

Results from the three hierarchical multiple regression analyses are presented in Table 2 . As hypothesized, r-BAS significantly and positively predicted PPI-Boldness psychopathic traits $(\beta=.51$, $p<.01)$, while the r-Freeze $(\beta=-.35, p<.01)$ subcomponent of the r-FFFS was a significant negative predictor. However, neither r-Fight, r-Flight, nor r-BIS were uniquely associated with PPIBoldness in this model. The overall model explained $44 \%$ of the variance in PPI-Boldness traits, $R^{2}=.44, F(7,279)=33.67, p<.001$.

Next, in a multiple regression analysis predicting PPIDisinhibition, our hypothesis was partially supported in that r-BAS $(\beta=.15, p<.01)$, r-Fight $(\beta=.28, p<.01)$, and r-Freeze $(\beta=.30, p<.01)$ all were positively and significantly associated with the outcome. Contrary to expectations, r-Flight negatively predicted PPI-Disinhibition $(\beta=-.17, p<.01)$, and r-BIS was unrelated to PPI-Disinhibition $(\beta=-.01, n s)$. The full model explained 
Table 1

Means, standard deviations, correlations, and alphas among Variables.

\begin{tabular}{|c|c|c|c|c|c|c|c|c|c|c|c|c|c|c|}
\hline Measure & 1 & 2 & 3 & 4 & 5 & 6 & 7 & 8 & 9 & 10 & 11 & 12 & 13 & 14 \\
\hline 1. PPI-R & $(.91)$ & & & & & & & & & & & & & \\
\hline 2. FD & $.71^{* *}$ & $(.89)$ & & & & & & & & & & & & \\
\hline 3. $\mathrm{SCI}$ & $.82^{* *}$ & $.22^{* * *}$ & $(.91)$ & & & & & & & & & & & \\
\hline 4. Coldheartedness & $.44^{* *}$ & $.23^{* *}$ & $.21^{* *}$ & $(.74)$ & & & & & & & & & & \\
\hline 5. PPI-Boldness & $.63^{* *}$ & $.95^{* *}$ & $.14^{*}$ & $.16^{* *}$ & $(.81)$ & & & & & & & & & \\
\hline 6. PPI-Meanness & $.52^{* *}$ & $.14^{*}$ & $.43^{* *}$ & $.89^{* *}$ & .06 & $(.76)$ & & & & & & & & \\
\hline 7. PPI-Disinhibition & $.66^{* *}$ & $.13^{*}$ & $.86^{* *}$ & $.12^{*}$ & .07 & $.27^{* * *}$ & $(.72)$ & & & & & & & \\
\hline 8. r-BAS & $.46^{* *}$ & $.51^{* *}$ & $.29^{* *}$ & -.02 & $.50^{* *}$ & -.02 & $.18^{* *}$ & $(.73)$ & & & & & & \\
\hline 9. r-BIS & $.24^{* *}$ & $.11^{* * *}$ & $.25^{* * *}$ & .05 & .07 & $.24^{* * *}$ & .11 & $.24^{* * *}$ & $(.62)$ & & & & & \\
\hline 10. r-FFFS & .01 & $-.31^{* *}$ & $.30^{* *}$ & $-.17^{* *}$ & $-.33^{* *}$ & -.01 & $.27^{* *}$ & .04 & $.22^{* *}$ & $(.68)$ & & & & \\
\hline 11. r-Fight & $.40^{* *}$ & .10 & $.46^{* *}$ & $.22^{* *}$ & .02 & $.33^{* *}$ & $.33^{* *}$ & $.12^{*}$ & $.23^{* *}$ & $.60^{* *}$ & $(.78)$ & & & \\
\hline 12. r-Flight & $-.32^{* *}$ & $-.32^{* *}$ & $-.15^{*}$ & $-.31^{* *}$ & $-.30^{* *}$ & $-.24^{* *}$ & -.08 & -.04 & .08 & $.66^{* *}$ & -.02 & $(.58)$ & & \\
\hline 13. r-Freeze & -.09 & $-.42^{* *}$ & $.26^{* *}$ & $-.26^{* *}$ & $-.40^{* *}$ & $-.14^{*}$ & $.28^{* *}$ & .00 & .11 & $.71^{* *}$ & $.13^{*}$ & $.32^{* * *}$ & $(.59)$ & \\
\hline 14. Age & $-.15^{* *}$ & -.04 & $-.18^{* *}$ & -.05 & -.04 & -.10 & $-.12^{*}$ & -.09 & $-.27^{* *}$ & $-.14^{*}$ & -.07 & .02 & $-.25^{* *}$ & - \\
\hline Mean & 282.29 & 112.74 & 139.77 & 29.77 & 58.39 & 33.08 & 35.00 & 12.25 & 12.64 & 53.04 & 17.36 & 17.92 & 17.76 & 21.33 \\
\hline Standard deviation & 35.9 & 18.89 & 24.03 & 6.28 & 10.56 & 6.76 & 6.55 & 3.12 & 3.01 & 7.74 & 4.23 & 3.82 & 3.68 & 5.90 \\
\hline Range & $189-405$ & $63-160$ & $89-214$ & $16-51$ & $32-87$ & $19-50$ & $19-60$ & $6-22$ & $6-25$ & $32-78$ & $6-28$ & $8-29$ & $7-28$ & $18-67$ \\
\hline
\end{tabular}

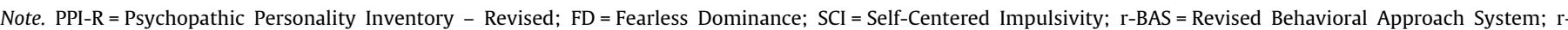

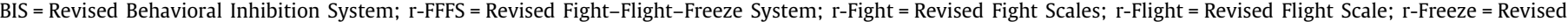
Freeze Scale.

Cronbach's $\alpha$ in parentheses along the diagonal.

${ }^{*} p<.05$.

$p<.01$.

Table 2

Hierarchical multiple regression analyses examining unique associations between PPI-R Triarchic scales and Revised Reinforcement Sensitivity Theory constructs.

\begin{tabular}{|c|c|c|c|c|c|c|}
\hline & \multicolumn{2}{|c|}{ PPI-Boldness } & \multicolumn{2}{|c|}{ PPI-Meanness } & \multicolumn{2}{|c|}{ PPI-Disinhibition } \\
\hline & $\beta$ & $t$ & $\beta$ & $t$ & $\beta$ & $t$ \\
\hline \multicolumn{7}{|l|}{ Step 1} \\
\hline Age & -.10 & $-2.07^{*}$ & -.07 & -1.26 & -.01 & -.25 \\
\hline Sex & -.16 & $-3.16^{* *}$ & -.04 & -.60 & -.01 & -.21 \\
\hline \multicolumn{7}{|l|}{ Step 2} \\
\hline r-BAS & .51 & $10.99^{* * *}$ & -.12 & $-2.13^{*}$ & .15 & $2.65^{* *}$ \\
\hline r-BIS & -.04 & -.78 & .21 & $3.59^{* *}$ & -.01 & -.20 \\
\hline r-Fight & -.03 & -.57 & .30 & $5.42^{* *}$ & .28 & $4.82^{* *}$ \\
\hline r-Flight & -.09 & -1.79 & -.20 & $-3.25^{* * *}$ & -.17 & $-2.71^{* * *}$ \\
\hline r-Freeze & -.35 & $-7.16^{* *}$ & -.15 & $-2.49^{*}$ & .30 & $5.02^{* * *}$ \\
\hline Adjusted $R^{2}$ & $.44^{*}$ & & $.22^{* *}$ & & $.19^{* *}$ & \\
\hline
\end{tabular}

Note. PPI-R = Psychopathic Personality Inventory - Revised; r-BAS = Revised Behavioral Approach System; r-BIS=Revised Behavioral Inhibition System; r-FFFS = Revised Fight-Flight-Freeze System; r-Fight = Revised Fight Scales; r-Flight $=$ Revised Flight Scale; r-Freeze = Revised Freeze Scale .

* $p<.05$.

$p<.01$.

$19 \%$ of the variance in PPI-Disinhibition, $R^{2}=.19, F(7,279)=10.82$, $p<.001$.

Finally, in the prediction of PPI-Meanness, the hypothesized relationship with r-FFFS was supported, as r-Fight positively and significantly predicted the outcome $(\beta=.30, p<.01)$, while r-Flight $(\beta=-.20, p<.01)$ and r-Freeze $(\beta=-.15, p<.05)$ were significant negative predictors. Contrary to hypotheses, PPI-Meanness was negatively predicted by r-BAS $(\beta=-.12, p<.05)$ and positively predicted by r-BIS $(\beta=.21, p<.01)$. The overall model accounted for $22 \%$ of the variance PPI-Meanness traits, $R^{2}=.22$, $F(7,279)=12.24, p<.001$

\section{Discussion}

The primary aim of the present study was to examine the associations of the three factors of the triarchic conceptualization of psychopathy with constructs of revised Reinforcement Sensitivity Theory (r-RST). Consistent with prior research, r-BAS sensitivity correlated positively with both PPI-Boldness and PPI-
Disinhibition at the bivariate level (Bijttebier et al., 2009; Sellbom \& Phillips, 2013). Associations between the triarchic constructs and r-FFFS were also largely consistent with expected results. Revised FFFS displayed a negative association with PPIBoldness and a positive association with PPI-Disinhibition. Moreover, subcomponents of r-FFFS demonstrated more nuanced associations. Revised Flight and r-Freeze were negatively associated with PPI-Boldness, while r-Fight and r-Freeze were positively associated with PPI-Disinhibition. Of note, r-FFFS was not associated with PPI-Meanness though each subcomponent demonstrated a significant association. Revised Fight was positively associated with PPI-Meanness, whereas r-Flight and r-Freeze were negatively associated. These findings highlight the importance of examining the subcomponents of the r-FFFS construct in relation to psychopathy factors.

With regard to r-BIS, results were not consistent with hypothesized relationships. PPI-Meanness was positively correlated with rBIS, which stands in stark contrast to previous research on o-BIS (Hall et al., 2014; Sellbom \& Phillips, 2013), as well as theoretical understandings of the meanness construct (Patrick et al., 2009). Furthermore, r-BIS was unrelated to PPI-Boldness and PPIDisinhibition, which again reflects a marked deviation from prior studies (Hall et al., 2014; Sellbom \& Phillips, 2013). These results should also be considered in light of the finding that r-BIS was positively associated with $r-B A S$ in this study $(r=.24, p<.01)$, an unexpected finding as these constructs are considered orthogonal (Jackson, 2009). Examination of the item content of the Jackson Five r-BIS scale suggests it may not adequately measure the experience of worry, anxious apprehension, and goal conflict described in r-RST (e.g., "I like my peers to know I am doing well;" Jackson, 2009). Instead, and consistent with the arguments of Smederevac et al. (2014), it appears that the items comprising the Jackson Five r-BIS scale may actually assess something akin to social competitiveness. This would clarify some of the curious findings in this study, as social competitiveness would be expected to positively correlate with PPI-Meanness due to the exploitative interpersonal style endemic to meanness. Similarly, the social poise and effectiveness characteristic of boldness traits may limit competitiveness in interpersonal situations (it simply may not be seen as necessary) and contribute to the nonsignificant association between PPIBoldness and Jackson Five r-BIS. 
The nonsignificant relationship between $\mathrm{r}$-BAS and PPIMeanness was also curious, given that excitement seeking is an attribute of this triarchic construct (Patrick et al., 2009). However, this unexpected finding may stem from the content coverage of the items that comprise PPI-Meanness. Specifically, PPIMeanness items do not appear to adequately capture the domain of excitement seeking, as they are derived from the Coldheartedness and Machiavellian Egocentricity subscales of the PPI-R (Hall et al., 2014). In the development of PPI-Meanness, it was found to be more closely related to a callous-unemotional interpersonal style with features of aggression and limited affiliation (Hall et al., 2014).

Multivariate findings shed additional light on the complex nature of these relationships. Overall, r-RST constructs appear important in understanding PPI-Boldness, explaining a substantial portion of the construct's variance. As anticipated, PPI-Boldness was positively predicted by r-BAS and r-Freeze after controlling for the remaining $r$-RST constructs and demographic variables. This finding again supports prior research that boldness psychopathic traits are characterized by an approach orientation and a relatively fearless temperament (Hall et al., 2014). Nineteen percent of the variance of PPI-Disinhibition was explained by r-RST constructs, with this factor characterized by increased r-BAS, r-Fight, and r-Freeze activation, and diminished r-Flight activation. These findings are consistent with a profile of increased negative affect and deficits in impulse control, while highlighting that overt escape is likely not a predominant response among those high in disinhibited psychopathic traits.

The constructs of r-RST explained $22 \%$ of the variance in PPIMeanness. Meanness was positively predicted by r-Fight and negatively predicted by r-Flight and r-Freeze, suggesting those high in meanness traits are less likely to escape or freeze in response to immediate threat, instead responding with aggression. Unlike boldness and disinhibition, PPI-Meanness exhibited a small negative association with r-BAS after controlling for other r-RST constructs and demographic variables, which again may reflect a lack of content coverage with regard to excitement seeking in the PPI-Meanness items. Also of note is the small to moderate positive association between r-BIS and meanness in the multivariate model. Again, the previously discussed questions regarding the construct validity of Jackson Five r-BIS may explain these unexpected findings.

Overall, results of the current study are supportive of prior contentions that Lykken's conceptualization of primary psychopathy may be characterized as "weak FFFS" under r-RST (Poythress et al., 2008), however our findings raise additional questions about the Jackson Five r-BIS scale and we are therefore unable to draw firm conclusions with regard to the relationship between r-BIS and the triarchic constructs of psychopathy.

\subsection{Strengths and limitations}

To our knowledge, the current study is the first to examine the triarchic conceptualization of psychopathy in relation to r-RST using a measure specifically designed to assess the revised theory. Indeed, the Jackson Five assessment of r-FFFS is more comprehensive than the BIS-fear subscale of the BIS/BAS Scales, and the ability to examine individual subcomponents of this system in relation to psychopathy is viewed as a significant strength. Several limitations must be considered however. Most notably, this study raises concerns regarding the construct validity of the Jackson Five r-BIS scale and additional research is necessary to further explicate this issue. Some subscales of the Jackson Five also exhibited low internal consistency (e.g., r-Flight and r-Freeze), however this may be a reflection of their limited number of items. Next, due to the recent development of the PPI-Triarchic scales, its validity is considered preliminary. Moreover, the PPI-Triarchic scales were developed using the original PPI and the present study utilized items from the PPI-R (however see Hall et al., 2014 for data suggesting veritable equivalence of the two forms). Also, this sample was limited to college students from one university in the northeastern area of the United States. While the sample was fairly diverse, this study was underpowered to examine potential racial and ethnic differences. Additional research with more diverse samples is necessary to assess the generalizability of these findings. Also, the majority of our sample was female, and while we statistically controlled for sex in regression analyses, future studies should examine the generalizability of findings to samples with more males. Lastly, the present study used a cross-sectional design, precluding any inferences regarding causality. Longitudinal research will allow for a better assessment of the role of r-RST in the development and maintenance of psychopathy over time.

\subsection{Conclusions and future directions}

Reinforcement Sensitivity Theory has been tremendously influential in furthering theory and research on the etiology of psychopathy. The significant changes to o-RST (Gray \& McNaughton, 2000) necessitate revisiting previous conceptualizations of psychopathy (e.g., the weak BIS hypothesis) under the revised framework. The current study demonstrated that while RST revisions add to our understanding of the etiology of psychopathy, the validation of purposefully-built r-RST measures remains as ongoing task. We encourage continued construct validation studies on the Jackson Five, as well as more recently developed r-RST measures such as the Corr-Cooper Reinforcement Sensitivity Theory Personality Questionnaire (RST-PQ; Corr \& Cooper, unpublished manuscript) and the Reinforcement Sensitivity Questionnaire (Smederevac et al., 2014).

\section{References}

Barlow, D. H. (2002). Anxiety and its disorders: The nature and treatment of anxiety and panic. New York: Guilford Press.

Bijttebier, P., Beck, I., Claes, L., \& Vandereycken, W. (2009). Gray's Reinforcement Sensitivity Theory as a framework for research on personality-psychopathology associations. Clinical Psychology Review, 29(5), 421-430.

Broerman, R.L., Ross, S.R., \& Corr, P.J. (2014). Throwing more light on the dark side of psychopathy: An extension of previous findings for the revised Reinforcement Sensitivity Theory.

Carver, C. S., \& White, T. L. (1994). Behavioral inhibition, behavioral activation, and affective responses to impending reward and punishment: the BIS/BAS scales. Journal of Personality and Social Psychology, 67(2), 319.

Cleckley, H. (1941). The mask of sanity. St. Louis, MO: Mosby.

Corr, P. J. (2008). Reinforcement sensitivity theory (RST): Introduction. In: P. J. Corr (Ed.), The Reinforcement Sensitivity Theory of Personality (pp. 1-43). Cambridge: Cambridge University Press.

Corr, P. J., \& Cooper, A. The Corr-Cooper Reinforcement Sensitivity Theory Personality Questionnaire (RST-PQ): Development and validation. Unpublished Manuscript, Retrieved on February 6, 2015 at http://www.philipcorr.net/uploads/ downloads/153.pdf.

Corr, P. J., DeYoung, C. G., \& McNaughton, N. (2013). Motivation and personality: A neuropsychological perspective. Social and Personality Psychology Compass, 7(3), $158-175$.

Drislane, L. E., Patrick, C. J., Sourander, A., Sillanmaki, L., Aggen, S. H., Elonheimo, H., et al. (2014). Distinct variants of extreme psychopathic individuals in society at large: Evidence from a population-based sample. Personality Disorders: Theory, Research, and Treatment, 5(2), 154-163.

Gray, J. A. (1970). The psychophysiological basis of introversion-extraversion. Behavior Research and Therapy, 8, 249-266.

Gray, J. A. (1982). The neurophysiology of anxiety: An inquiry into the functions of the septal-hippocampal system. Oxford: Oxford University Press.

Gray, J. A., \& McNaughton, N. (2000). The neuropsychology of anxiety: An enquiry into the functions of the septo-hippocampal system. New York: Oxford University Press.

Hall, J. R., Drislane, L. E., Patrick, C. J., Morano, M., Lilienfeld, S. O., \& Poythress, N. G. (2014). Development and validation of triarchic construct scales from the Psychopathic Personality Inventory. Psychological Assessment, 26(2), 447-461.

Harnett, P. H., Loxton, N. J., \& Jackson, C. J. (2013). Revised Reinforcement Sensitivity Theory: Implications for psychopathology and psychological health. Personality and Individual Differences, 54(3), 432-437. 
Heym, N., Ferguson, E., \& Lawrence, C. (2008). An evaluation of the relationship between Gray's revised RST and Eysenck's PEN: Distinguishing BIS and FFFS in Carver and White's BIS/BAS scales. Personality and Individual Differences, 45(8), 709-715.

Hughes, K. A., Moore, R. A., Morris, P. H., \& Corr, P. J. (2012). Throwing light on the dark side of personality: Reinforcement sensitivity theory and primary/ secondary psychopathy in a student population. Personality and Individual Differences, 52(4), 532-536.

Jackson, C. J. (2009). Jackson-5 scales of revised reinforcement sensitivity theory ( $\mathrm{r}$ RST) and their application to dysfunctional real world outcomes. Journal of Research in Personality, 43(4), 556-569.

Karpman, B. (1941). On the need of separating psychopathy into two distinct clinical types: The symptomatic and the idiopathic. Journal of Criminal Psychopathology, 3, 112-137.

Lilienfeld, S. O., \& Andrews, B. P. (1996). Development and preliminary validation of a self-report measure of psychopathic personality traits in noncriminal populations. Journal of Personality Assessment, 66(3), 488-524.

Lilienfeld, S. O., \& Widows, M. R. (2005). Psychopathic Personality Inventory - Revised professional manual. Lutz, FL: Psychological Assessment Resources Inc.

Lykken, D. T. (1995). The antisocial personalities. Psychology Press.

Newman, J. P., MacCoon, D. G., Vaughn, L. J., \& Sadeh, N. (2005). Validating a distinction between primary and secondary psychopathy with measures of Gray's BIS and BAS constructs. Journal of Abnormal Psychology, 114(2), 319-323.

Patrick, C. J., Fowles, D. C., \& Krueger, R. F. (2009). Triarchic conceptualization of psychopathy: Developmental origins of disinhibition, boldness, and meanness. Development and Psychopathology, 21(03), 913-938.
Poythress, N. G., Skeem, J. L., Weir, J., Lilienfeld, S. O., Douglas, K. S., Edens, J. F., et al (2008). Psychometric properties of Carver and White's (1994) BIS/BAS scales in a large sample of offenders. Personality and Individual Differences, 45(8), $732-737$.

Ross, S. R., Molto, J., Poy, R., Segarra, P., Pastor, M. C., \& Montañés, S. (2007). Gray’s model and psychopathy: BIS but not BAS differentiates primary from secondary psychopathy in noninstitutionalized young adults. Personality and Individua Differences, 43(7), 1644-1655.

Sellbom, M., \& Phillips, T. R. (2013). An examination of the triarchic conceptualization of psychopathy in incarcerated and nonincarcerated samples. Journal of Abnormal Psychology, 122(1), 208-214.

Skeem, J. L. Poythress, N. Edens, J. F, Lilienfeld, S, O. \& Cale, E. M. (2003). Psychopathic personality or personalities? Exploring potential variants of psychopathy and their implications for risk assessment. Aggression and Violent Behavior, 8(5), 513-546.

Smederevac, S., Mitrović, D., Čolović, P., \& Nikolašević, Ž. (2014). Validation of the measure of revised Reinforcement Sensitivity Theory constructs. Journal of Individual Differences, 35(1), 12-21.

Smillie, L. D. Pickering A. D. \& Jackson, C. J. (2006). The new Reinforcement Sensitivity Theory: Implications for personality measurement. Personality and Social Psychology Review, 10(4), 320-335.

White, T. L., \& Depue, R. A. (1999). Differential association of traits of fear and anxiety with norepinephrine-and dark-induced pupil reactivity. Journal of Personality and Social Psychology, 77(4), 863. 